\title{
Participação de um agente adrenérgico não peptídico na ação inotrópica positiva da fração polar da Bryopsis pennata
}

\author{
Participation of a non-peptide adrenergic agent on positive inotropic effect of the polar \\ fraction of Bryopsis pennata
}

\author{
Andrea Lucia Natali OLIANI ${ }^{1}$; Jeanete Lopes NAVES ${ }^{1}$; José Carlos de FREITAS ${ }^{1}$; Claudio Alvarenga \\ OLIVEIRA $^{2}$
}

${ }^{1}$ Instituto de Biociências da Universidade de São Paulo, São Paulo-SP

${ }^{2}$ Faculdade de Medicina Veterinária e Zootecnia da Universidade de São Paulo, São Paulo-SP

\begin{abstract}
Resumo
As algas marinhas representam uma rica fonte de compostos bioativos, algumas delas precursoras de ferramentas farmacológicas e de substâncias potencialmente úteis para o desenvolvimento de novos fármacos. A macroalga Bryopsis pennata, Cloroficea pertencente à ordem Caulerpales, sin. Bryopsidales é uma espécie tropical encontrada em diversos costões rochosos. Essa espécie produz uma defesa química tóxica para organismos herbívoros e potencial de se tornar invasiva e dominante em condições ambientais favoráveis. Este trabalho é uma investigação do efeito cardiotônico da fração polar da B. pennata .O cultivo, em ambiente controlado destituído de contaminantes, foi realizado no intuito de comparar seus efeitos com os efeitos da alga coletada. O efeito cardiotônico cronotrópico positivo em tiras ventriculares de anuros ficou fortemente evidenciado nos testes com frações polares de algas coletadas e cultivadas, não apresentando diferenças significativas entre as frações. O propranolol antagonizou o efeito cardiotônico e enzimas endopeptidases não reagiram com a fração polar da $B$. pennata. Testes bioquímicos demonstraram que a fração com efeito cardiotônico é de caráter ácido e apresenta peso molecular menor que 10.000 daltons.
\end{abstract}

Palavras-chave: Bryopsis pennata. Inotrópico positivo. Propranolol. Alga medicinal. Princípio ativo.

\begin{abstract}
Marine algae are a rich source of bioactive compounds and some of them have shown to be useful for the development of new pharmacological tools and medicines. Bryopsis pennata, (Clorofícea, Caulerpales, sin. Bryopsidales) is a marine algae that can be found in the Southeastern Brazilian coast and elsewhere. The species produces a toxic chemical defense to the herbivorous organisms and possesses a potential to become invasive and dominant in favorable environmental conditions. The present study is about the investigation of the cardiotonic effect of the polar extract of $B$. pennata. The cultive in laboratory without any contaminants was made to compare studies performed with the collected algae extracts and the ones cultivated effect. The cardiotonic effect in the anurou's ventricular strips was strongly unequivocal when it was tested with the polar extracts from the collected and cultivated algae. The propranolol antagonized polar effects of these algae and the endopeptidase enzyme did not change the effects of polar extracts of Bryopsis pennata. Biochemical tests showed that the polar fraction presenting the inotropic cardiac activity weighs less than 10.000 Daltons and has an acid character.
\end{abstract}

Keywords: Bryopsis pennata. Inotropic positive. Propranolol.

\section{Introdução}

Os primeiros registros do uso terapêutico, indicador empírico da presença de substâncias bioativas em algas, estão documentados num compêndio médico chinês de 2700 anos a.C. entre os povos do leste da Ásia. A medicina popular oriental utiliza amplamente
Correspondência para:

Andrea Lucia Natali Oliani

R. Dr. Hélio Fidelis, 121, bl 03, apto 53. CEP 05351-035 - Butantã São

Paulo-SP, F. (11) 2935-7845, (11) 7656-8497,

e-mail: andrea.natali@gmail.com

Recebido para publicação: 21/07/2009

Aprovado para publicação: 07/01/2010 
as algas como tratamentos para várias enfermidades tais como verminoses, gota, nefrite e outras ${ }^{1}$.

Os avanços científicos na farmacologia marinha têm revelado diversos compostos bioativos, ampliando as possibilidades de aplicação das algas como fonte direta de medicamentos ou inspirando a síntese de novas substâncias a partir das estruturas moleculares descobertas. Investigações sugerem que a função primária dos metabólitos secundários das algas é deter a herbivoria, porém não se exclui a possibilidade de outras funções e atividades detectadas em ensaios laboratoriais ${ }^{2,3,4}$.

A presença de toxinas em diversas algas tem interesse para a saúde pública, pois as mesmas podem provocar envenenamentos em populações que utilizam algas em sua dieta. Outras espécies, porém, aparentemente não tóxicas, funcionam como complemento nutricional há algum tempo em países consumidores de algas, devido ao seu alto teor de vitaminas e iodo. O problema do bócio endêmico, que atinge determinadas populações no mundo, poderia ser grandemente diminuído pela exploração das algas, as quais são portadoras de alto teor de iodo, da ordem de 100 a 40.000 vezes maior que a quantidade existente na água do mar ${ }^{5}$.

Se considerarmos que, no mundo inteiro, muitos indivíduos morrem de distúrbios circulatórios, mais do que de qualquer outro tipo de doença, essa seria uma forte razão para que aumentássemos substancialmente as pesquisas farmacológicas a respeito de substâncias de algas marinhas, para fomentar a produção de drogas antiateroscleróticas ${ }^{4,6}$.

A ação antibiótica de muitos extratos de algas, inclusive a atividade contra vírus, bactérias, leveduras, fungos e microalgas, está bem estabelecida assim como atividades anticoagulante, antilipêmica, antitumoral, hipocolesterolêmica, entre outras. A laminina, isolada da alga Laminaria angustula previne a hipertensão em ratos e provoca a redução na força dos batimentos cardíacos, efeito inotrópico negativo segundo Freitas e Marsiglio ${ }^{7,8}$. A espécie Ulva pertusa também apresentou efeito inotrópico negativo9 ${ }^{9}$. Considerando a relevância das algas para os estudos farmacológicos, Marques et al. ${ }^{10}$ alertam que ao lado da continuidade dos levantamentos florísticos deve ser dada ênfase também a outras pesquisas, tais como aquelas sobre a estrutura das comunidades e extração de produtos naturais em gêneros promissores como Laurencia, $\mathrm{Di}$ genea, Dictyota, Bryopsis e Codium, que podem sofrer influências ecológicas, potencializando ou inibindo (alterando), as sínteses de metabólitos secundários.

Com potencial para se tornar invasiva, a macroalga Bryopsis pennata (ordem Caulerpales), é uma espécie tropical encontrada em diversos oceanos. Além de não sofrer ação predatória de herbívoros marinhos, produz defesa química tóxica, promove alelopatia e se reproduz facilmente a partir de pequenos fragmentos. O extrato da Bryopsis pennata, em diferentes épocas do ano, provocou o aumento no tônus muscular com ação inotrópica e cronotrópica positiva em coração de anuro. Relatou-se também a presença de dois componentes ativos no extrato da alga coletada, não identificados, nas frações do extrato bruto da $B$. pennata, os quais possuem efeitos neurotóxicos reversíveis em nervo sensorial isolado de crustáceo e inotrópico e cronotrópico positivos em coração de anuros (Rana Catesbeiana $)^{11}$.

A iniciativa do estudo de novas substâncias oriundas do ambiente marinho vem sendo manifestada pela equipe do Laboratório de Produtos Naturais de Origem Marinha do Instituto de Biociências da USP, onde abordam de maneira multidisciplinar os desafios da investigação e o desenvolvimento da farmacologia marinha. Biólogos, veterinários, químicos, bioquímicos e farmacologistas, reunidos em torno desse tema, tentam equacionar esforços distintos em cada fase da pesquisa. A observação dos aspectos ecológicos, as coletas, a extração, a análise in vitro do extrato e sua posterior purificação para identificação da substância ativa vêm dependendo desses esforços multidisciplinares. 
Neste trabalho, estudamos a alga verde Bryopsis pennata, sob o ponto de vista farmacológico, e sua ação cardiotônica em coração de anuro. Utilizando preparações biológicas e determinando, até onde nos foi possível, o efeito comparativo da alga cultivada com a alga coletada no coração de anuros, testes bioquímicos foram realizados para elucidar o princípio ativo, além da resposta farmacológica diante de ensaio cardiotônico com propranolol.

\section{Material e Método}

As macroalgas foram coletadas na Praia do Tombo, Guarujá, Litoral Sul do Estado de São Paulo, em Setembro de 2005 e 2006. Após lavagem em água do mar esterilizada, uma parte das algas foi congelada e a outra foi levada ao Laboratório de Cultura de Algas do Instituto de Botânica de São Paulo, transportadas em frascos plásticos. O cultivo foi realizado com água do mar enriquecida com meio Von Stosch, salinidade 30-32 $\mu$ ps, temperatura $21 \pm 2{ }^{\circ} \mathrm{C}$, irradiância 40,0 $60,0 \mu \mathrm{mol}$ fótons. m-2. s-1, fotoperíodo $14 \mathrm{~h}$ luz, $10 \mathrm{~h}$ escuro.

As macroalgas foram quantificadas, homogeneizadas com metanol (1:10 m:v) e filtradas. O metanol foi evaporado a vácuo $\left(50\right.$ a $\left.60{ }^{\circ} \mathrm{C}\right)$; a suspensão aquosa contendo clorofila foi filtrada em papel filtro de celulose centrifugado (Fanem Excelsa II 206 ${ }^{\circ}$ ) a $5040 \mathrm{G}$ unid/10 minutos. O precipitado foi descartado e o sobrenadante foi novamente submetido à evaporação a vácuo para obtenção de novo resíduo. O resíduo final (fração polar) foi diluído em água destilada e mantido a $20^{\circ} \mathrm{C}$ negativos.

Foram preparadas Frações Polares de Bryopsis pennata (FPBp) cultivadas e coletadas, separadamente.

A FPBp foi incubada com tripsina e quimiotripsina $(1 \mathrm{mg} / \mathrm{mL})$ durante $15,60,90$ e 120 minutos a $37^{\circ} \mathrm{C} \mathrm{e}$, a seguir, testada na tira ventricular de anuro em uma cuba de $10 \mathrm{ml}$. A concentração final foi de $0,2 \mathrm{mg} / \mathrm{ml}$ da FPBp tratado com tripsina e com quimiotripsina.
Teste Cardiotônico - Tiras ventriculares isoladas de coração de rãs da espécie Rana Catesbeiana foram utilizadas neste ensaio. As rãs foram sedadas com éter, e em seguida foram submetidas à espinhalação na coluna cervical. Não foi utilizado nenhum outro anestésico, de modo a evitar que seus efeitos interferissem na elucidação dos resultados farmacológicos esperados. A técnica seguiu rigorosamente as regras estipuladas pelo COBEA (Colégio Brasileiro de Experimentação Animal). A preparação foi realizada em uma cuba com 10 mL de solução de Ringer (NaCI 111,2; KCI 1,4; $\mathrm{CaCI}_{2} 1,0 ; \mathrm{NaH}_{2} \mathrm{PO}_{4} 0,1 ; \mathrm{NaHCO}_{3} 2,4$ e $\mathrm{C}_{6} \mathrm{H}_{12} \mathrm{O}_{6}$ $11,1)$, acoplada a uma unidade isoladora de estímulo e um Estimulador (GRASS ${ }^{\mathrm{TM}}$ ). O ápice da tira ventricular foi conectado a um transdutor isométrico (Narco Biosystems, F-50) e a parte basal fixada a um gancho de vidro entre dois eletrodos de prata clore$\operatorname{tada}^{11}$. Administramos pulsos de 0,2 ms de duração, $0,2 \mathrm{~Hz}$ de frequência em voltagem supramáxima de $100 \mathrm{v}$ (monofásico). As respostas musculares foram isometricamente registradas em um fisiógrafo (Narco Biosystem, MK-II SER). A tensão utilizada foi de 0,5 g. Foram testadas quatro diferentes concentrações da FPBp coletada e cultivada $(25 \mu \mathrm{g} / \mathrm{mL}, 50 \mu \mathrm{g} / \mathrm{mL}, 0,1$ $\mathrm{mg} / \mathrm{mL}$ e $0,2 \mathrm{mg} / \mathrm{mL}$ ).

O ensaio também foi realizado com o propanolol, bloqueador não seletivo dos receptores adrenérgicos $\beta 1$ e $\beta 2$. Utilizamos a concentração de $0,2 \mu \mathrm{M}$ de propranolol na cuba de $10 \mathrm{~mL}$ contendo FPBp na concentração de 0,2 mg/mL.

Para caracterizar as substâncias envolvidas na resposta cardiotônica, a FPBp foi dialisada em tubo de ultrafiltração de 10.000 Daltons. A FPBp também foi estudada quanto ao seu caráter ácido/base, utilizando-se uma coluna de troca iônica Amberlite (IRA-400 AR, USA).

\section{Resultados}

Teste Cardiotônico - Os resultados mostram que substâncias presentes na FPBp cultivada e também na 
coletada $(n=6)$ apresentam um efeito inotrópico positivo reversível em tiras ventriculares de anuros (Figuras 1 e 2). A ação é dose-dependente quando comparada, em porcentagem, ao efeito cardiotônico sem adição da FPBp. A $\mathrm{CE}_{50}$ foi de $1,118 \mathrm{mg} / \mathrm{mL}$ (Figura 3).

O propanolol (0,2 uM), menor dose que apresentou efeito, adicionado à preparação do teste cardiotônico após a adição da FPBp $(0,2 \mathrm{mg} / \mathrm{mL})$, concentração de máxima atividade miocontrátil, antagonizou completamente o efeito inotrópico positivo após cerca de 40 segundos (Figuras 4 e 5).

Avaliação da natureza da FPBp - dialisamos a amostra e constatamos que a fração ativa tem peso molecular menor que 10.000 Dáltons. A fração dialisada

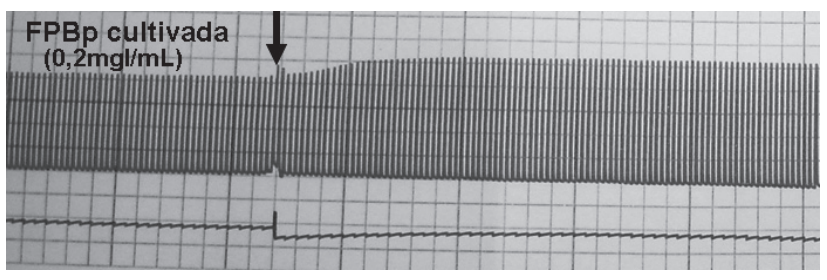

Figura 1 - Resultado do efeito farmacológico cardiotônico da alga cultivada, realizado em tiras ventriculares de anuros. Observa-se na seta o início do efeito inotrópico positivo da fração polar da alga $B$. pennata cultivada na concentração de $0,2 \mathrm{mg} / \mathrm{mL}$

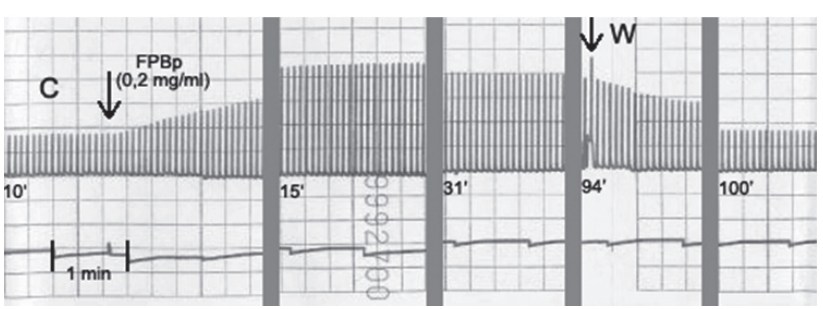

Figura 2 - Resultado do efeito farmacológico cardiotônico da alga coletada, realizado em tiras ventriculares de anuros. Observa-se em "C" a atividade cardiotônica registrada na tira ventricular sem ação farmacológica. A primeira seta indica o momento da adição da Fração Polar da Bp (FPBp - 0,2 mg/mL), e nos segundos seguintes o aumento progressivo da atividade inotrópica, onde se evidencia uma contínua atividade positiva durante cerca de 80 minutos. Após a lavagem da amostra ("W"), o efeito inotrópico positivo se torna completamente reversível, atingindo a mesma amplitude do início do experimento em apenas seis minutos foi submetidia ao tratamento com as enzimas tripsina e quimiotripsina e, após esse tratamento, verificamos que estas não produziram nenhuma alteração na ação miocontrátil (Figuras 6 e 7).

A atividade farmacológica do extrato bruto só é evidenciada quando submetida à fração ácida da amostra (Figura 8), o que não ocorre com a fração de caráter básico (Figura 9), determinando dessa forma um caráter ácido para a fração potencialmente ativa.

\section{Discussão}

Os resultados mostram que substâncias presentes em B. pennata apresentam um efeito inotrópico positivo no coração de anuro corroborando um estudo

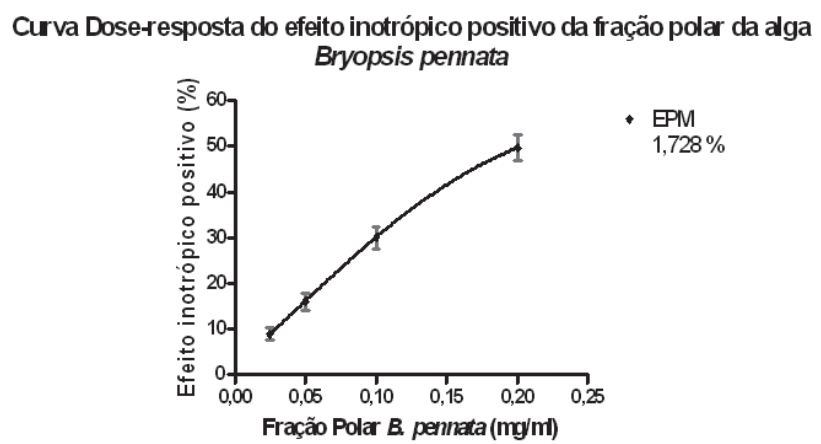

Figura 3 - Curva dose-resposta do efeito inotrópico positivo da fração polar da alga Bryopsis pennata realizado nas doses $25 \mu \mathrm{g} / \mathrm{mL}, 50$ $\mu \mathrm{g} / \mathrm{mL}, 0,1 \mathrm{mg} / \mathrm{mL}$ e $0,2 \mathrm{mg} / \mathrm{mL}$. N $=6 "$ EC50 $=1.118 \mathrm{mg} / \mathrm{mL}-$ IC $95 \%=0,3581 \mathrm{a}$ $3,493 \mathrm{mg} / \mathrm{mL}$

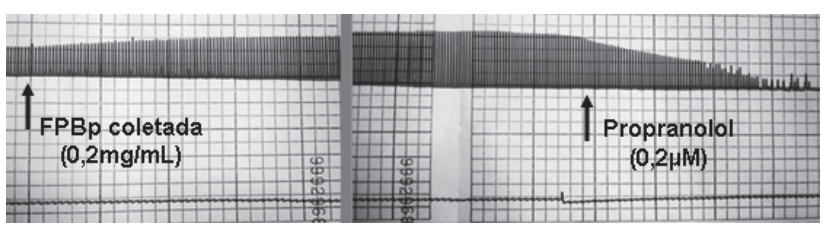

Figura 4 - A figura mostra, na primeira seta, o resultado do efeito farmacológico cardiotônico da alga coletada, realizado em tiras ventriculares de anuros na dose de $0,2 \mathrm{mg} / \mathrm{mL}$. Após cerca de 60 minutos de atividade cardiotônica máxima da FPBp, administrou-se, na segunda seta, dose única de $0,2 \mu \mathrm{M}$ do propranolol, evidenciando após 40 segundos, seu efeito bloqueador total sob a ação da fração polar da alga $B$. Pennata 


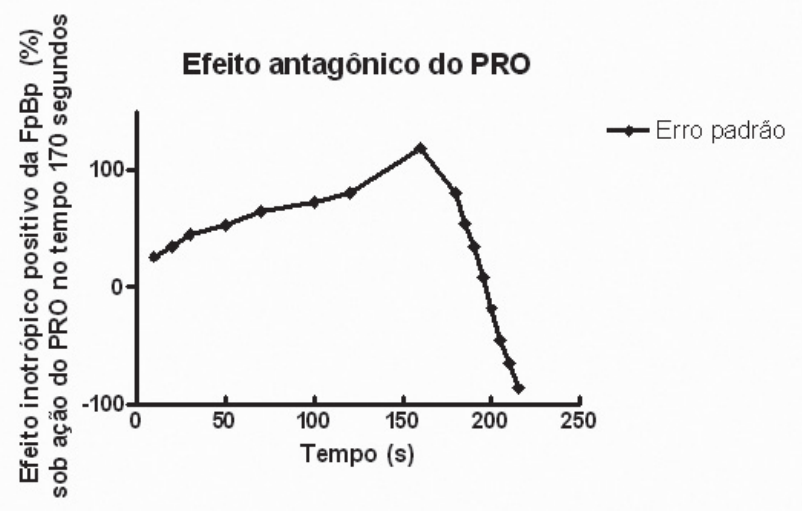

Figura 5 - Resultado do efeito farmacológico cardiotônico da alga coletada, realizado em tiras ventriculares de anuros na dose de $0,2 \mathrm{mg} / \mathrm{mL}$. 150 segundos após a administração da FPBp, administrouse o propranolol $(0,2 \mu \mathrm{M})$. Após cerca de 70 segundos sob efeito do propranolol promoveuse total efeito bloqueador na atividade cardiotônica da tira ventricular de anuro

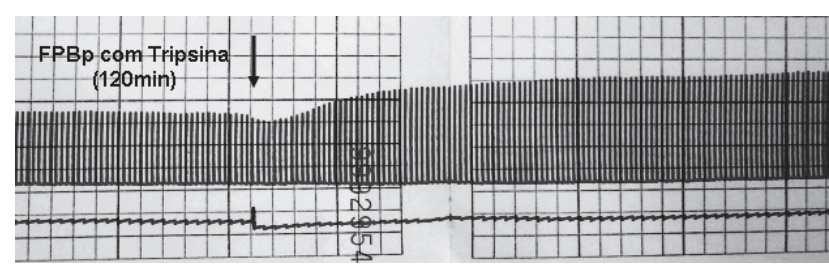

Figura 6 - Efeito inotrópico positivo da FPBp coletada $(0,2 \mathrm{mg} / \mathrm{mL})$ tratada durante 120 minutos com a endopeptidase tripsina, evidenciando a preservação do seu efeito farmacológico mesmo na presença da enzima

anterior realizado com a mesma espécie coletada em São Sebastião $(\mathrm{SP})^{11}$ e contrastando com a espécie Ulva pertusa que tem efeito inotrópico negativo ${ }^{9}$. Verificamos que não houve diferença nas respostas cardiotônicas entre as frações polares de algas cultivadas e coletadas, mostrando que o cultivo não alterou a produção dos metabólitos da alga e que os possíveis contaminantes da alga coletada não interferiram nas respostas farmacológicas.

A quimiotripsina é uma endopeptidase que quebra diversas ligações peptídicas entre resíduos do lado $\mathrm{C}$ terminal dos aminoácidos fenilalanina, triptofano e tirosina ${ }^{1}$. Se o substrato em estudo, substâncias da macroalga B. pennata, não foi sensível ou

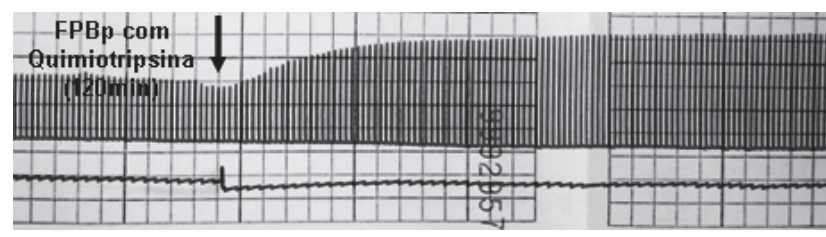

Figura 7 - Efeito inotrópico positivo da FPBp coletada $(0,2$ $\mathrm{mg} / \mathrm{mL}$ ) tratada durante 120 minutos com a endopeptidase quimiotripsina, evidenciando a preservação do seu efeito farmacológico mesmo na presença da enzima

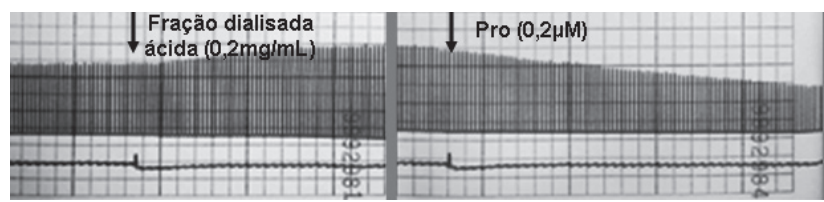

Figura 8 - A figura mostra, na primeira seta, o efeito inotrópico positivo da Fração Polar de caráter ácido $(0,2 \mathrm{mg} / \mathrm{mL})$. Após alguns minutos, administrou-se o propranolol $(0,2 \mu \mathrm{M})$, onde se evidenciou o efeito antagônico na ação cardiotônica da fração dialisada

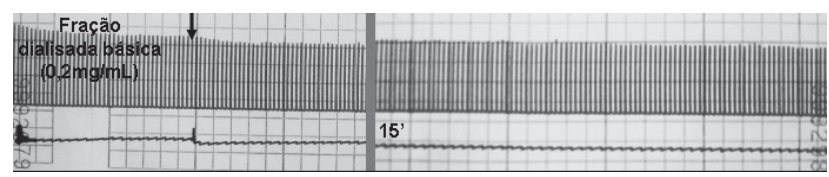

Figura 9 - A figura mostra que não ocorre efeito inotrópico positivo da Fração Polar de caráter básico $(0,2$ $\mathrm{mg} / \mathrm{mL}$ ) em momento algum

degradado por essas enzimas, isso indica fortemente que ela não é de natureza proteica. Nesse caso, podemos sugerir que seja um peptídeo ativo com poucas ligações, ou uma substância de natureza química não peptídica.

A justificativa para a associação entre a fração polar de B. pennata e o propanolol foi tentar elucidar seu provável mecanismo de ação já que o propranolol é um $\beta$-bloqueador não seletivo, que bloqueia receptores $\beta 1$ e $\beta 2$ adrenérgicos, impedindo assim a ação da noradrenalina nesses receptores ${ }^{1}$. A adição in vitro desse bloqueador antagonizou o efeito inotrópico positivo da fração polar da B. pennata. Dessa forma, o aumento de inotropismo induzido pela FPBp na tira 
ventricular de anuro estimulada eletricamente, poderia ser explicado por uma ação despolarizante nas terminações nervosas que chegam ao coração, via terminais noradrenérgicos.

\section{Conclusão}

A atividade cardiotônica da fração polar é causada por compostos com peso molecular menor que 10.000 Daltons, de caráter ácido e natureza não proteica. $\mathrm{O}$ cultivo não alterou a produção dos compostos ativos testados neste trabalho.

\section{Referências}

1. RANG, H. P.; DALE M. M.; RITTER, J. M.; MOORE, P. K. Farmacologia. 5. ed. Rio de Janeiro: Elsevier, 2003. 904 p.

2. ARAGÃO, V. Métodos de análise de conteúdo estomacal. Estudo de caso: Acanthurus coerulus (Bloch \& Schneider, 1801) - Teleostei, Acanthuridae - na Reserva Biológica do Atol das Rocas. 2003. 72 f. Dissertação (Mestrado em Biologia Marinha). Universidade Federal Fluminense, Niterói, 2003.

3. BARBOSA, J. P.; TEIXEIRA, V. L.; VILLAÇA, R.; PEREIRA; ABRANTES, J. L.; FRUGULHETTI, C. P. P. A dolabellane diterpene from the brazilian brown alga Dictyota pfaffii. Biochemical Systematics and Ecology, v. 31, n. 12, p. 14511453,2003

4. RANGEL, M. Estudos farmacológicos do extrato da esponja marinha Geodia corticostylifera (Porifera, Desmospongiae). 1999. 98 p. Dissertação (Mestrado) - Universidade de São Paulo, Instituto de Biociências, São Paulo, 1999.

5. FREITAS, J. C.; SATO, S.; OGATA, T.; KODAMA, M. Guanidine neurotoxins are released with the digestive fluid of crabs (crustacean, brachyura). Toxicon, v. 33, n. 2, p. 201-208, 1994.

6. NAVES J. L.; PRADO M. P.; RANGEL M.; SANCTIS, B.; MACHADO-SANTELI, G.; FREITAS, J. C.Citotoxicity in
A ação inotrópica positiva foi reversível em tiras ventriculares de anuro. Esse efeito pode ser bloqueado por propranolol, o que indicaria uma ação indireta da fração, ou seja, atuando via receptores noradrenérgicos.

\section{Agradecimentos}

À Profa. Dra. Nair S. Yokoya do Instituto de Botânica - SP, ao Laboratório de Cultura de Algas "Marilza Cordeiro Marino" - Seção de Ficologia, ao CNPq, pelo financiamento junto ao Instituto de Botânica e bolsa de produtividade em pesquisa, à CAPES e à FUNDAMAR.

the marine dinoflagellate Prorocentrum mexicanum from Brazil. Comparative Biochemistry and Physiology, v. 143, n. 1, p. 73-77, 2006.

7. FREITAS, J. C.; MARSIGLIO, A. F. Pharmacological activity of extracts of some marine algae from the Brazilian coast. Boletim Fisiologia Animal, São Paulo, v. 10, p. 61-68, 1986.

8. FREITAS, J. C. Efeitos comportamentais de neurotoxinas guanidínicas em meio aquático. Revista de Etologia, v. 1, n. 2, p. 117-126. 2000.

9. YAMADA, K.; SHIZURI, Y.; ISHIDA, Y.; SHIBATA, S. Cardiac inhibitory action of constituents of the marine green alga Ulva pertusa. Journal of Pharmaceutical Sciences, v. 72, n. 8, p. 1912-1995, 1983.

10.MARQUES, L. V.; VILLAÇA, R. C.; PEREIRA, S. M. B.; CRESPO, R. Susceptibility of macroalgae to herbivorous fishesat rocas atoll. Brasil. Botanica Marina Berlin, v. 49, p. 379-385, 2006.

11.SAKAMOTO, M. M. Ações farmacológicas do extrato de alga marinha Bryopsis pennata (Chlorophyta, Caulerpales). 1993. 124 p. Tese (Doutorado) - Instituto de Ciências Biomédicas, Universidade de São Paulo, São Paulo, 1993. 